\title{
Morphology Study of Si Surface Topography Irradiated by Ar Ion Beam Sputtering
}

\author{
HONGMEI BI, YING LIANG ${ }^{*}$ and BO WANG \\ Science Department, Heilongjiang Bayi Agricultural University, China \\ chemistry412@126.com
}

Received 15 February 2015 / Accepted 4 March 2015

\begin{abstract}
In this paper, Si crystal (000) surface was bombarded by Ar ion beam, with the energy of $90 \mathrm{KeV}$ and the irradiating time of $10 \mathrm{~min}$. In the process of irradiation, there were bulges on the surface of Si crystal surface, the highest of which reached $100 \mathrm{~nm}$. The information of roughness (such as change in level, angle and etc.) were studied by AFM, TEM, SEM and Raman.
\end{abstract}

Keywords: Silicon, Ar ion beam, Roughness

\section{Introduction}

Silicon is one of the popular materials in semiconductor industry; it is widely used in the semiconductor devices. Amorphous Si thin film is a burgeoning semiconductor film, it has developed quickly since $70 \mathrm{~s}$ in twentieth century. While, amorphous Si has fatal defects which restricts its application; it has short lifetime and low photoelectric conversion efficiency. In these years, the two defects have been improved a lot. Compared with the $\mathrm{Si}$ crystal materials, amorphous Si materials are of lower cost which would take more part in the Si materials market in the future.

Nowadays amorphous $\mathrm{Si}$ is usually prepared by plasma chemical vapor deposition. The irradiation with energetic ion beams is also a good choice to prepare micro-nanostructure, the irradiated samples has characteristic mechanical, electric, optical and thermal properties $^{1,2}$. In the radiation process, there will be swelling phenomenon with low-energy ion beams $\mathrm{s}^{3-5}$ and can be accompanied by changes in the topography with creation of $\mathrm{Si}$ crystalline and amorphous Si. Under different irradiation conditions this surface erosion can result in well-ordered nanostructures. Of the crystalline semiconductors, some researchers considered that the swelling phenomena are mainly contributed by amorphization ${ }^{3}$. This swelling effect has been obtained in a great number of different semiconductors and insulators such as $\mathrm{Si}, \mathrm{Ge}, \mathrm{SiC}, \mathrm{GaAs}, \mathrm{GaN}, \mathrm{MgO}, \mathrm{LiNbO} 3, \mathrm{Al} 2 \mathrm{O} 3, \mathrm{LiF}$, etc., which is related with ion mass, fluence, energy, dwell time, angle $e t c^{6}$.

In this work, we study the swelling phenomenon of Si sample irradiated by Ar beam. The swelling height and the disorder of Si crystal are measured by AFM, TEM, SEM and the appearance of Si crystalline and amorphous Si are detected by Raman spectrum. 


\section{Experimental}

Single-crystal $\mathrm{Si}(000)$ samples of $0.5 \mathrm{~mm}$ with an area of $1 \times 1 \mathrm{~cm}^{2}$ were used to observe the swelling experiment in this study. Before irradiation, all of the samples were cleaned by HF acid and then soaked in ultra-pure water. An ion beam machining apparatus (10-GHz NANOGAN) was applied, the pressure of the vacuum chamber was in the range of $6.0 \times 10^{-6}$ $\sim 7.0 \times 10^{-6} \mathrm{~Pa}$ at RT with a fixed energy of $90 \mathrm{KeV}$.

The surface roughness information was observed by atomic force microscope (AFM, S II nanotechnology Inc. SPI 3800N). SEM photographs were observed on a XL-30-ESEMFEG with an operating voltage varying from 5 to $20 \mathrm{KeV}$. TEM was employed by JEOL2100 electron microscope. Raman spectra were collected at ambient temperature on a Jobin Yvon HR 800 micro -Raman spectrometer.

\section{Results and Discussion}

Raman spectrum was employed to explore the inner structure of samples, it was of great meaning in the field of nanotechnology, micro-nanofabrication, modification and machining. $\mathrm{Si}$ crystal samples were irradiated by $\mathrm{Ar}$ ion beam in the fluence range of $0.5 \times 10^{15} \sim$ $8 \times 10^{16} / \mathrm{cm}^{2}$. In Figure $1,520 \mathrm{~cm}^{-1}$ peak was attributed to the characteristic peak of Si crystal; $480 \mathrm{~cm}^{-1}$ peak was attributed to the amorphous Si layer, which was enhanced after irradiation.
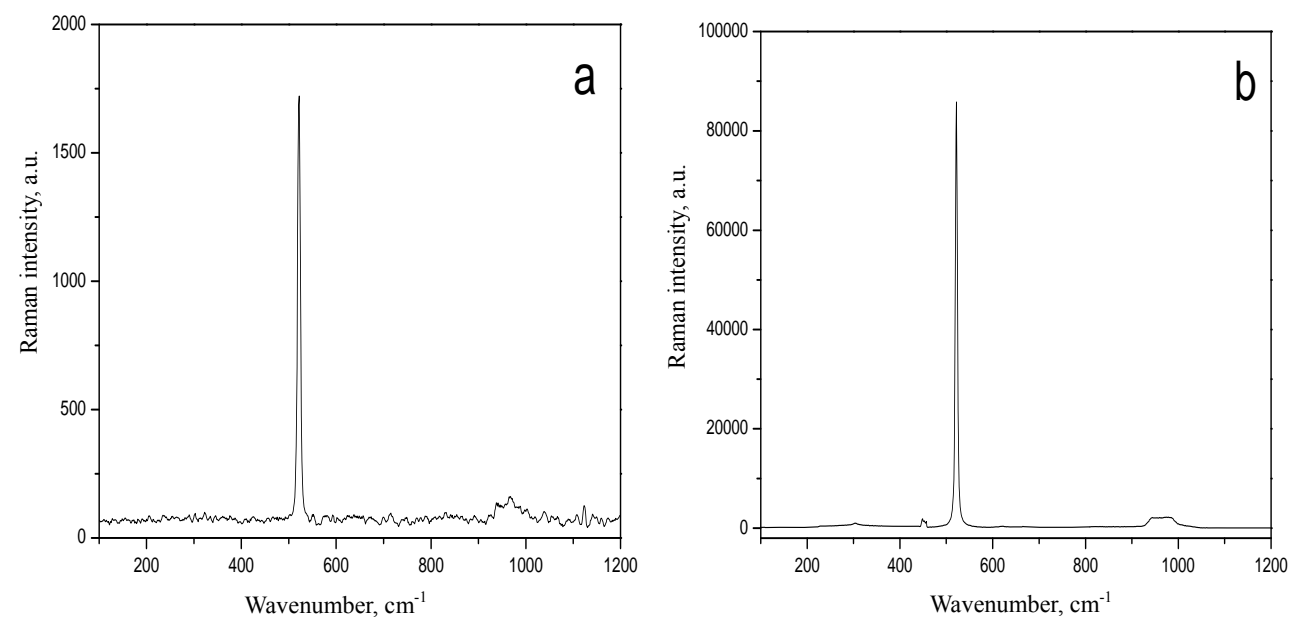

Figure 1. Raman spectra of Si crystal samples: a) before irradiation; b) after irradiation

In Raman spectra, 420-490 $\mathrm{cm}^{-1}$ was attributed to the LO phonon peak of amorphous $\mathrm{Si} ; 490-510 \mathrm{~cm}^{-1}$ was related with the situation of Si crystalline grain, which was due to the $\mathrm{O}$ atom influenced on the original angle of Si material, i.e there was defect on the Si surface; $510-520 \mathrm{~cm}^{-1}$ was related with the optical phonon of Si crystalline grain. When the size of Si crystalline grain reduced to the nanoscale, it would red-shift as the of Si size decreasing.

The surface and roughness of Si could be seen from Figure 2 and Figure 3 (white part in Figure 3 was the irradiation zone). The thickness of amorphous Si was about $100 \mathrm{~nm}$. That was to say that in the irradiation process $\mathrm{Si}$ crystalline changed into amorphous $\mathrm{Si}$. It was a new method of preparing nanostructure. 


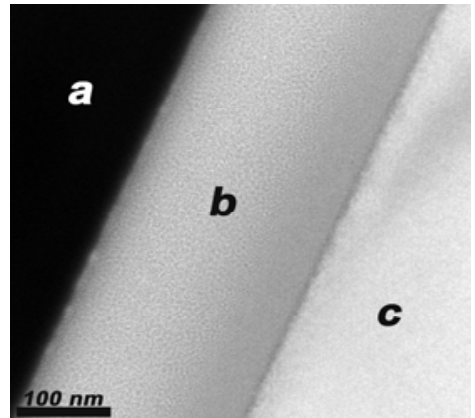

Figure 2. TEM morphology and roughness of irradiated Si sample part a:Pt base; part b: amorphous $\mathrm{Si}$; part c: Si crystal

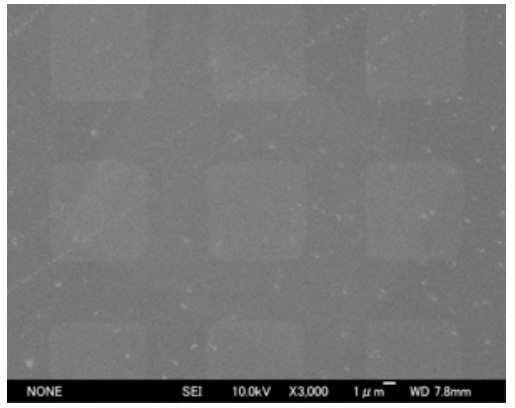

Figure 3. SEMtopography image of irradiated Si sample

The information of AFM indicated the morphology of the Si crystalline surface layer, where a disordered crystalline layer caused non-uniformity of the etching process. In Figure 4, the height of amorphous Si had been increased by $7 \mathrm{~nm}$.

In the irradiation of Ar ion beam, there was roughness on the Si surface with the creation of amorphous $\mathrm{Si}$ and $\mathrm{Si}$ crystalline grain. The irradiated samples were characterized by AFM, SEM, TEM and Raman, the thickness of amorphous Si was about $100 \mathrm{~nm}$.

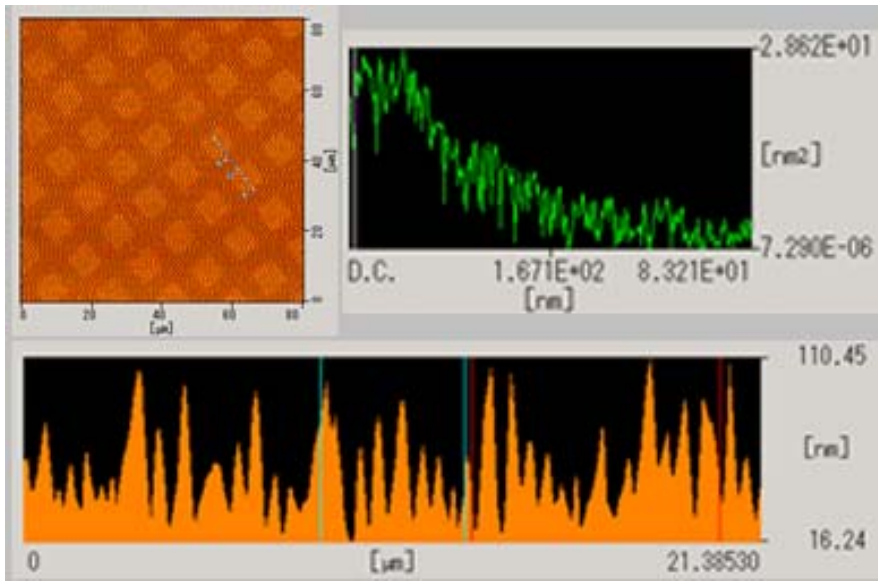

Figure 4. AFMtopography image of Si surface

\section{References}

1. Kolíbal M, Matlocha T, Vystavěl T and Šikola T, Nanotechnology, 2011, 22(10), 105304; DOI:10.1088/0957-4484/22/10/105304

2. Rota A, Contri S, Gazzadi G, Cottafava S, Gualtieri E and Valeri S, Sur Sci., 2006, 600(18), 3718-3722;

3. Giri P K, Raineri V, Franzo G and Rimini E, Phys Rev B, 2001, 65, 012110.

4. Wittmaack K, Int J Mass Spectrom., 2008, 269(1-2), 24-33.

5. $\quad$ Giri P, Mater Sci Eng B, 2005, 121(3), 238-243.

6. Ziberi B, Cornejo M, Frost F and Rauschenbach B, J Phys Condens Matter., 2009, 21(22), 224003; DOI:10.1088/0953-8984/21/22/224003 